\title{
Application of photodynamic therapy for liver malignancies
}

\author{
Heng Zou ${ }^{1 \#}$, Fusheng Wang ${ }^{2 \#}$, Jiang-Jiao Zhou ${ }^{1}, \mathrm{Xi} \mathrm{Liu}{ }^{1}$, Qing He ${ }^{1}$, Cong Wang ${ }^{1}$, Yan-Wen Zheng ${ }^{1}$, \\ Yu Wen ${ }^{1}$, Li Xiong ${ }^{1}$
}

${ }^{1}$ Department of General Surgery, The Second Xiangya Hospital, Central South University, Changsha 410011, China; ${ }^{2}$ Department of General Surgery, Fuyang People's Hospital, Fuyang 236000, China

Contributions: (I) Conception and design: L Xiong; (II) Administrative support: Y Wen; (III) Provision of study materials or patients: Q He; (IV) Collection and assembly of data: H Zou, X Liu; (V) Data analysis and interpretation: C Wang; (VI) Manuscript writing: All authors; (VII) Final approval of manuscript: All authors.

"These authors contributed equally to this work.

Correspondence to: Dr. Li Xiong, MD. Department of General Surgery, The Second Xiangya Hospital, Central South University, Changsha 410011, China. Email: lixionghn@csu.edu.cn.

\begin{abstract}
Liver malignancies include primary and metastatic tumors. Limited progress has been achieved in improving the survival rate of patients with advanced stage liver cancer and who are unsuitable for surgery. Apart from surgery, chemoradiotherapy, trans-arterial chemoembolization and radiofrequency ablation, a novel therapeutic modality is needed for the clinical treatment of liver cancer. Photodynamic therapy (PDT) is a novel strategy for treating patients with advanced cancers; it uses a light-triggered cytotoxic photosensitizer and a laser light. PDT provides patients with a potential treatment approach with minimal invasion and low toxicity, that is, the whole course of treatment is painless, harmless, and repeatable. Therefore, PDT has been considered an effective palliative treatment for advanced liver cancers. To date, PDT has been used to treat hepatocellular carcinoma, cholangiocarcinoma, hepatoblastoma and liver metastases. Clinical outcomes reveal that PDT can be considered a promising treatment modality for all liver cancers to improve the quality and quantity of life of patients. Despite the advances achieved with this approach, several challenges still impede the application of PDT to liver malignancies. In this review, we focus on the recent advancements and discuss the future prospects of PDT in treating liver malignancies.
\end{abstract}

Keywords: Photodynamic therapy (PDT); photosensitizer; liver malignancies; new modality for cancer

Submitted Dec 31, 2019. Accepted for publication Feb 14, 2020.

doi: 10.21037/jgo.2020.02.10

View this article at: http://dx.doi.org/10.21037/jgo.2020.02.10

\section{Introduction}

Liver cancer, which primarily includes hepatocellular carcinoma (HCC), cholangiocarcinoma, or mixed carcinoma, originates in the liver and intrahepatic bile duct. Liver cancer is the second leading cause of cancer death worldwide, following lung cancer, with a remarkably high mortality-to-incidence rate of up to over 0.95 , according to statistics (1). Currently, surgical resection is the most efficient and mainstay treatment for liver cancer. Other treatment modalities, such as radiofrequency ablation (RFA), trans-arterial chemoembolization, percutaneous ethanol injection, percutaneous microwave coagulation therapy, radiotherapy, and systemic chemotherapy, have been introduced for liver cancer treatment (2-4). Although considerable advancement in liver cancer treatment modalities has been attained, many patients with unresectable liver malignancies still suffer from a low survival rate. Therefore, it is meaningful to discover new therapeutic agents, cancer detection methods, and novel treatment modalities under these situations.

Nanoparticles (NPs) gain much popularity due to biological, therapeutic and medical applications in modern times (5). Iqbal et al. synthesized silver oxide NP via chemical aqueous method and found it could inhibit the cell viability of HepG2 cell line effectively (6). To improve the 
distinguishing ability of normal cells from tumor cells, Atif et al. used fluorescence spectroscopy to discriminate normal and malignant cell lines and found considerably different spectral features between normal melanocytes and malignant cells like Wish, MCF-7, and HepG2 (7). Photodynamic therapy (PDT), also known as photochemotherapy, is a procedure that uses photosensitizing drugs along with specific wavelength of light to trigger reactions and generate cytotoxic reactive oxygen species (ROS), such as singlet oxygen and free radicals, to treat various tumors. PDT is officially approved by the United States Food and Drug Administration as a drug-device combined therapy for cancers, and it has been applied to the treatment of various solid tumors, such as brain tumor, rhabdomyosarcoma and esophageal, breast, bladder, liver, cervical, larynx, and colorectal cancers (8-12).

The biological mechanism of PDT is complicated. After intravenous or oral applications, the photosensitizer predominantly concentrates in tumor tissues and subsequently is irradiated with a light of appropriate wavelength (13). Following absorbing photons, the photosensitizer transforms from its ground singlet state into an excited singlet state, which is followed by intersystem crossing to an excited triplet state. The triple state either can undergo a type I reaction, which is oxygen independent and reacts with an organic molecule directly to form free radicals, or a type II reaction in which the excess energy is transferred to molecular oxygen $\left(\mathrm{O}_{2}\right)$, leading to the formation of singlet oxygen $\left({ }^{1} \mathrm{O}_{2}\right)(14,15)$. These two reactions lead to the production of ROS, which causes fatal damage to neoplastic tissues $(16,17)$. PDT-induced stress triggers a number of cellular responses, such as apoptosis, necrosis, and/or autophagic cell death, in cancer cells $(18,19)$. In addition to directly killing cancerous cells, PDT can inhibit tumor growth by destroying the vasculature associated with the tumor and influencing the tumor cell cycle (20). Interestingly, PDT can initiate or improve immune responses, such as the secretion of cytokines, leukocyte chemoattractants, growth factors, and other regulators. This process can lead to infiltration of tissues with neutrophils, macrophages, mastocytes, and natural killer cells (21). An interactive function among each of these parts ultimately drives the photochemical reaction (22).

PDT is a minimally invasive therapeutic modality and features several advantages when applied to cancer treatment (15). First, photocytotoxic reactions occur only within pathological tissues where the photosensitizer accumulates, thereby enabling selective destruction.
Second, PDT is a painless and simple method that can be used for outpatients. Third, PDT can be applied along with other palliative therapies, such as chemotherapy and radiotherapy (23). Although PDT has been proven to be an effective clinical therapy for cancers such as liver malignancies, its utility has been limited for several reasons: (I) the absorption of specific photosensitizers may be greater in normal liver tissues than in liver malignant tumors, thereby reducing the sensitivity and specificity of this therapy for liver tumor; (II) as liver has a large volume and pigmented tissues, the shallow penetration of light affect the phototoxicity of PDT (13); (III) because of the deep location of liver in the abdominal cavity, it is difficult for the transmission of laser fiber to the tumor. Rapid progress has been made by research groups in photosensitizer modification, light source improvements, and new drug delivery development to solve these problems and facilitate the application of PDT to liver tumors.

In this review, we will summarize the contemporary practices of PDT for liver malignancies.

\section{PDT for HCC}

HCC accounts for most liver cancer cases, posing a severe global public health problem (2). Surgical resection by removal of cancerous tissues offers the best long-term outcome in HCC patients, but curative therapeutic option is limited for patients with advanced or terminal HCC. Thus, a new therapeutic method is urgently needed.

PDT has been demonstrated as an effective method for treating HCC (24-28). Multiple mechanisms are involved in PDT-mediated tumor cell killing for HCC in vitro and vivo. To measure the mechanism of cell toxicity of PDT on liver cancer cell lines, Shi R and his colleagues (29) used sinoporphyrin sodium as a photosensitizer toward human HCC, which included bel7402 and HepG2 cell lines. The experiment indicated that PDT might be induced by injury to the mitochondria, which then initiated apoptotic responses, such as cytochrome c release into the cytoplasm and caspase protein activation. Tang et al. (30) demonstrated that with pheophorbide a as a photosensitizer, PDT on human HCC can inhibit multi-drug resistance by downregulating the expression of $\mathrm{P}$-glycoproteins via c-Jun $\mathrm{N}$-terminal kinase activation in vitro. They also noted that PDT treatment mediated by pheophorbide a was more efficient in inhibiting the growth of Hep3B cells than that of the normal hepatic cell line WRL-68; PDT possibly targeted the mitochondria, which were more active in 
cancer cells than in normal cells, to generate ROS near the mitochondrial membrane (31). As a result, the altered potential of the mitochondrial membrane led to the release of cytochrome $\mathrm{c}$ from the mitochondria to the cytoplasm and initiated apoptosis. Another programmed cell death pathway, autophagy, can exert cytoprotective function when apoptosis occurs during PDT (32). Recently, Domagala et al. reported that the inhibition of autophagy could sensitize Hela and MCF-7 cancer cell lines to photofrin-based PDT (33). However, whether the same mechanism exists in HCC cell lines remains unknown. Therefore, further studies are needed to clarify the role of autophagy in PDT for HCC. PDT was also found to be linked with immune responses. Zhang and his colleagues (34) established a disease model by injecting a H22 cell suspension into Kunming mice; they observed that an increase in the population of $\mathrm{CD}^{+}, \mathrm{CD}^{+}$, and $\mathrm{CD} 19^{+}$cells was linked to the tumor growth inhibition after administering the PDT-generated vaccine. They reported that PDTgenerated vaccines could be used as an adjuvant therapy for cancer. PDT could also trigger inflammatory responses in addition to promoting apoptosis. It was reported that hypericin-mediated PDT can cause the death of HepG2 cells by regulating the expression of apoptosis-associated genes, such as caspases and cytochrome complex. Also, a remarkable increase of interleukin-6 was observed, whose expression level showed a close link with the apoptosis of tumor cells and caspase activity (27).

Positive surgical margin is an important reason for the recurrence of HCC after surgery (35). Intraoperative PDT is used as a concomitant and adjuvant therapy that can kill possible residual tumor cells in the surgical margin of liver to reduce the possibility of tumor recurrence. Muragaki et al. reported a phase II clinical study on intraoperative PDT with talaporfin sodium and semiconductor laser in patients with malignant parenchymal brain tumors (36). The 12-month overall survival and 6-month progression-free survival of the included 22 patients reached $95.5 \%$ and $91 \%$, respectively. The authors concluded that intraoperative PDT may be a potentially effective and sufficiently safe option for adjuvant treatment of malignant brain tumors. However, no other similar studies have reported the intraoperative PDT of HCC. With the development of laparoscopic instruments and surgical techniques, PDT for HCC can be conducted during minimally invasive operations (Figure 1A), that is, laparoscopic-assisted PDT. The laser can be transmitted to the lesions through a $12 \mathrm{~mm}$ diameter trocar. Two main endoscope technologies exist for laparoscopic-assisted PDT (37): laser fiber separating from the endoscope and the combination of optical fiberscope and laser fiber. The latter integrates the two parts, and is the ideal instrument to develop in the future for laparoscopicassisted PDT. However, one disadvantage of laparoscopicassisted PDT is that the laser irradiation field is difficult to control, possibly injuring the surrounding normal tissues, and thereby causing serious complications.

\section{PDT for cholangiocarcinoma}

Cholangiocarcinoma, a rare malignancy originating from epithelial cells of the biliary tree, remains the second most common primary liver cancer, following HCC (38). Cholangiocarcinoma is relatively difficult to diagnose given its deep location and the lack of definitive diagnostic criteria (39). Most patients present inoperable cholangiocarcinoma at the time of diagnosis, and their survival is measured in months (median survival: around 5 months) (40). Long survival time is only associated with a R0 margin in resection, whereas the five-year survival rate reaches $30-40 \%$ after curative resection $(41,42)$. Therefore, palliative treatment is needed for most cholangiocarcinoma patients.

PDT is an effective palliative method for advanced bile duct carcinoma (43-47). The first randomized controlled trial (RCT) to study the effect of PDT on nonresectable cholangiocarcinoma was reported in 2003 (48). The research compared cholangiocarcinoma patients treated by PDT and stent with those who received a stent only. The results suggested that the combination of PDT and stent offered better treatment outcomes for nonresectable cholangiocarcinoma than stent only. Another RCT was conducted for 32 patients with nonresectable bile duct cancer in 2005 (49). In the PDT group, photosan-3 (R) at a dosage of $2 \mathrm{mg} / \mathrm{kg}$ body weight was administrated, whereas the control group was treated with endoprostheses. The median survival time of the control group was 7 months, whereas that of the PDT group was significantly longer at 21 months $(\mathrm{P}<0.05)$. These RCT studies provide high-quality evidence for the use of PDT in patients with cholangiocarcinoma.

Chemotherapy is an important treatment modality for cholangiocarcinoma (50). The combination of chemotherapy with PDT for cholangiocarcinoma can theoretically obtain better treatment effect than PDT alone. Nonaka et al. (51) reported that the combination therapy of PDT with gemcitabine and oxaliplatin showed synergic 

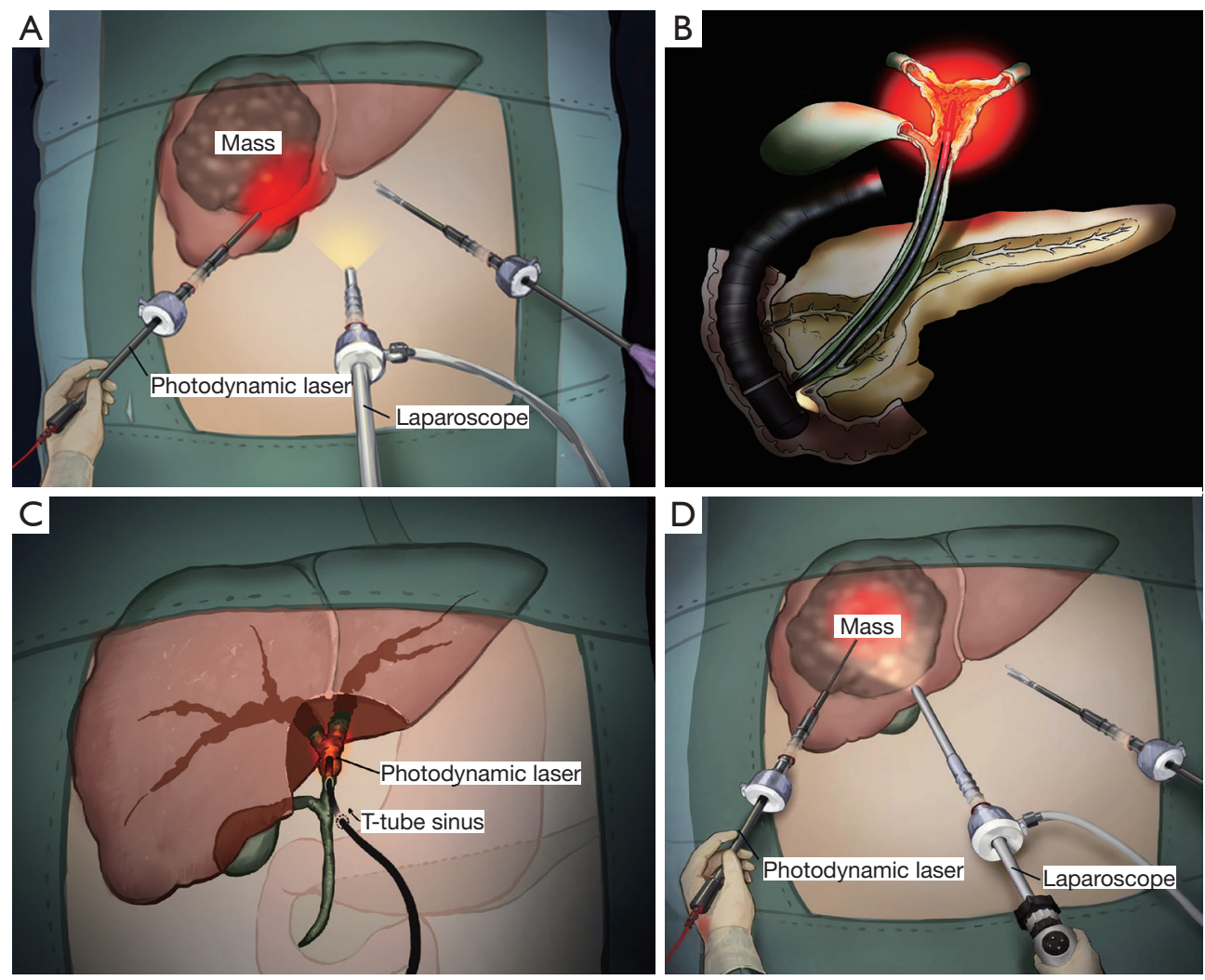

Figure 1 Illustrations of PDT for liver malignancies. (A) Laparoscopic-assisted photodynamic therapy (PDT) for hepatocellular carcinoma; (B) endoscopic retrograde cholangiopancreatography-directed PDT for cholangiocarcinoma; (C) PDT for cholangiocarcinoma through the T-tube sinus; (D) interstitial PDT for metastatic liver cancer.

effects on necrosis, apoptosis, and cytostatic alterations in advanced bile duct carcinoma. A randomized phase II trial reported by Park et al. (52) revealed that PDT plus S-1 was associated with better overall survival and progressionfree survival than PDT alone in patients with unresectable hilar cholangiocarcinoma. Another retrospective study enrolled 74 patients with hilar cholangiocarcinoma (53). Among these patients, 16 were treated with PDT and gemcitabine with or without cisplatin, whereas 58 were treated with PDT only. The results showed that PDT with chemotherapy achieved a longer survival time than PDT alone. Wentrup et al. (54) reviewed 68 patients with hilar nonresectable cholangiocarcinoma treated with either PDT plus chemotherapy or PDT monotherapy. They observed that the PDT plus chemotherapy group achieved a significantly higher one-year survival rate than the PDT monotherapy group $(\mathrm{P}=0.001)$. In general, the tumoricidal effect of PDT is often limited to the inner 4-6 mm of the tumor wall (55). However, most cholangiocarcinomas are locally advanced when diagnosed, and the lesions may be thick. Meanwhile, micrometastasis or distant metastasis may exist for advanced cholangiocarcinoma. PDT, which primarily plays a role in local regions, may feature limited therapeutic effects for patients with such conditions. Therefore, systemic chemotherapy may be used to obtain survival benefit for the patients with cholangiocarcinoma receiving PDT.

Biliary drainage can be beneficial as a palliative treatment in patients with unresectable cholangiocarcinoma, with longer survival time and less cost than surgical treatment (56). Leggett et al. (57) used meta-analysis to compare the overall outcome and effectiveness of biliary stenting combined with PDT with that of biliary stenting therapy alone in patients with cholangiocarcinoma. They revealed that the palliative treatment of cholangiocarcinoma with PDT is associated with survival benefits, that is, the improved biliary drainage and quality of patients' life. The following meta-analysis conducted in 2015 obtained similar 
results (58). Another meta-analysis that included 10 studies discovered survival periods of 413.04 and 183.41 days in PDT plus biliary stenting group and biliary stenting only group, respectively (59). The author concluded that PDT plus stenting is superior to stenting alone and recommended PDT as an adjunct to biliary stenting in patients with unresectable cholangiocarcinoma.

When performing PDT on cholangiocarcinoma, endoscopic retrograde cholangiopancreatography (ERCP) and percutaneous transhepatic cholangioscopy (PTCS) are two common delivery methods for laser radiation (60) (Figure $1 B$ ). Both approaches are minimally invasive, and they feature a low incidence of adverse events. A recent study reported that the overall survival after PTCS- or ERCP-directed PDT showed no statistically difference (11.6 versus 9.5 months, $\mathrm{P}=0.96$ ) in patients with advanced hilar cholangiocarcinoma (61). ERCP-directed PDT presents several advantages (61): (I) multiple segments can be treated in one time; (II) compared with PTCS-directed PDT, ERCP-directed PDT requires no waiting time for sinus tract maturation to allow the passage of cholangioscopy. However, limitations also exist for ERCP-directed PDT: (I) the appropriate location for placement of the fiber and probe used in PDT can be difficult to determine because extrahepatic bile duct carcinoma spreads longitudinally (62); (II) ERCP-directed PDT cannot accurately evaluate the response to treatment because it is performed under fluoroscopy guidance; (III) the patients may hesitate in accepting the peroral transpapillary approach; (IV) ERCPdirected PDT can more likely cause serious complications, such as acute pancreatitis and hematobilia, than PTCSdirected PDT. Meanwhile, given that PTCS-directed PDT is performed using direct endoscopic visual control, it can provide effective, visual, and homogenous irradiation of the targeted lesion, and it can be monitored repeatedly without periodic peroral endoscopy. Nevertheless, PTCS-directed PDT also presents drawbacks: (I) the PTBD tube should be left in place for a long time until the final PDT session to maintain the patency of the PTBD tract, thereby causing inconvenience to patients; (II) the PTBD procedure may cause peritoneal dissemination of cancer cells in the bile. In addition to ERCP and PTCS, laser fibers can also be introduced through the T-tube sinus for patients receiving laparotomy (Figure 1C), which can be convenient for patients to receive repeated PDT. In summary, PDT offers an important option for patients with cholangiocarcinoma, especially the unresectable type.

\section{PDT for hepatoblastoma}

Hepatoblastoma is the most common primary liver cancer in infants and children, accounting for around $1.5 \%$ of all incidence of pediatric malignancies (24). A low long-term survival rate is observed in patients with unresectable advanced hepatoblastoma (63). Hypericin, a photosensitizer derived from the natural products of flowering plants belonging to Hypericum (64), exhibits a stable fluorescence, desirable tissue penetration, preferential tumor retention, and greater cytotoxic effects in tumor cells than in normal tissues (65). This photosensitizer shows potential application in PDT for hepatoblastoma because it causes no severe side effects (66). Seitz et al. (24) demonstrated that the enhancement of concentration of hypericin in two hepatoblastoma cell lines, namely, HuH6 and HepT1, resulted in decreased tumor cell viability. Future studies are needed to establish the use of hypericinPDT for hepatoblastoma in vivo. Bergmann et al. (67) used 5-aminolevulinic acid (5-ALA) as a photosensitizer and demonstrated a marked and specific fluorescence in human hepatoblastoma $(\mathrm{HuH} 6)$ in vitro. They also observed that human hepatoblastoma was susceptible to PDT in a nude rat model. Curcumin is a yellow-orange dye derived from the rhizome of Curcuma longa. This compound is one of the most extensively studied phytochemicals. Curcumin could facilitate the absorption of radiation between 350 $500 \mathrm{~nm}$ and cause oxygen-dependent phototoxicity $(68,69)$. Curcumin-mediated PDT could enhance the anti-tumor properties of curcumin in hepatoblastoma cell lines (HuH6 and HepT1) by inducing the loss of viability via ROS production (70).

In general, surgical resection offers the mainstay of therapy for hepatoblastoma. For unresectable cases, chemotherapy is an important palliative treatment that significantly improves the overall survival period (71). Thus, PDT offers an alternatively effective method for treating hepatoblastoma, although further in vivo studies are needed.

\section{PDT for metastatic liver cancer}

Metastatic liver cancer, also known as secondary liver cancer, mostly develops from colorectal cancer. The median survival time of patients with colorectal liver metastases is 6-9 months if untreated (72), whereas the rate of operable colorectal liver metastases only reaches $10 \%$ in all patients (73). To date, surgical removal of metastatic tumor 
is the only curative treatment method. Other treatments, such as RFA, chemoradiotherapy, and PDT, are alternative modalities. PDT may be specifically suitable for patients with unresectable liver metastases or with liver metastases in the vicinity of large vessels.

Some findings indicate that PDT for metastatic liver cancer can achieve satisfactory effects $(13,74,75)$. Interstitial PDT (IPDT) is a good choice for deeply seated tumors or tumors thicker than $10 \mathrm{~mm}$ (76) (Figure 1D). IPDT involves the insertion of laser fibers directly into the tumors. The laser fibers can be inserted via needles, or placed in catheters (76). Light can be delivered through the end of the fibers, or through a fiber with a cylindrical diffuser end. To evaluate the IPDT for the treatment of solid liver tumors, researchers used LS 11 (talaporfin sodium with an applied dose of $40 \mathrm{mg} / \mathrm{m}^{2}$ ) as a photosensitizer to treat four metastatic liver cancers of four patients (three colorectal carcinoma and one melanoma). The LS 11 was activated via CT-guided percutaneously inserted intratumoral Lumaflex Light Sources. The mean diameter of tumor necrosis was around $14 \mathrm{~mm}$ (range, 13 to $17 \mathrm{~mm}$ ), and no cutaneous phototoxicity was observed (77). Bacteriochlorin a (BCA) is a nontoxic photosensitizer derived from bacteriochlorophyll a, and early researchers have discovered its preferential retention in certain types of tumors. Subsequently, a study reported that the BCA concentrations in normal liver and tumor of a Wag/Rij rat model implanted with colon carcinoma CC531 cells failed to show tissue preferences after the intravenous injection of BCA $(10 \mathrm{mg} / \mathrm{kg})$ and interstitial irradiation of $760 \mathrm{~nm}$ laser light (78). The author also revealed that the concentration of BCA showed no significant fluctuation but declined rapidly in the first 4 h. This finding implied that optimal results with IPDT could be obtained by illumination within a short interval after the administration of BCA (78). Another experiment used Photofrin for IPDT in a rat liver metastasis model, with the colon carcinoma CC531 cells implanted into the Wag/Rij rat model. The results confirmed that IPDT using Photofrin as a photosensitizer could cause the major destruction of tumor tissues with minimal liver damage (79). The main advantage of IPDT is that the light delivery from multiple fibers enables the treatment of large and deep tumors, which cannot be illuminated with external beam PDT (76).

Altogether, these findings provide the novel aspects of PDT modality in the treatment of metastatic liver cancer.

\section{Photosensitizers for anti-liver malignant tumors}

The modifications of PDT are focused on the type of photosensitizer, the intensity and wavelength of light controlling the tumor tissue penetration, the light delivery devices, and the interval between the administration of photosensitizer and irradiation (80). The most currently used photosensitizers exhibit absorption peaks at the 600-750 nm range in experimental and clinical PDT for liver malignancies (81). Given the large volume of liver, the PDT triggered by long-wavelength light is meaningful for the treatment of liver cancer. Several methods that can improve light delivery and intensity have been proposed for the treatment of deep cancers (82). The first method involves the development of photosensitizers activated by near-infrared (NIR) light, which presents a stronger tissue penetration than ultraviolet or visible light. The second method uses upconversion nanoparticles (UCNPs), which can be excited at NIR and then emit light in the ultravioletvisible range for PDT activation (83). Liang et al. (84) combined a new photosensitizer of KillerRed with UCNPs to obtain photosensitizing bio-nanohybrids, the KillerRedUCNPs, which exhibited excellent colloidal stability in biological buffers and low cytotoxicity in the dark. They demonstrated that the KillerRed-UCNPs exhibited superiority over unbound KillerRed in vitro PDT model of MDA-MB-231 cells buried under $\sim 1$-cm pork tissue. The third method is the use of $\mathrm{X}$-ray-induced scintillation or afterglow NPs for PDT activation (85).

Thus far, various photosensitizers such as hematoporphyrin derivative (26), pheophorbide a (86), ALA (87), sinoporphyrin sodium (29), and indocyanine green (88) have been evaluated and validated in clinical practice for the treatment of liver malignancies. Normally, an optimized photosensitizer is highly soluble and shows low toxicity before illumination and can produce maximum phototoxic effect in a restricted area around the tumor cells but not in the adjacent normal tissues (89).

NP-mediated targeted drug delivery system is a novel PDT approach that increases the tumor specificity of photosensitizer for liver malignancies. Tumor angiogenesis is a process in which new blood vessels in tumor tissues form from the existing ones. The tumor vasculature substantially differs from normal tissue blood vessels. The defective vascular architectures for tumors include discontinuous endothelial lining, lack of smooth muscle 
Table 1 Summary of active targeting for photodynamic therapy on liver malignancies

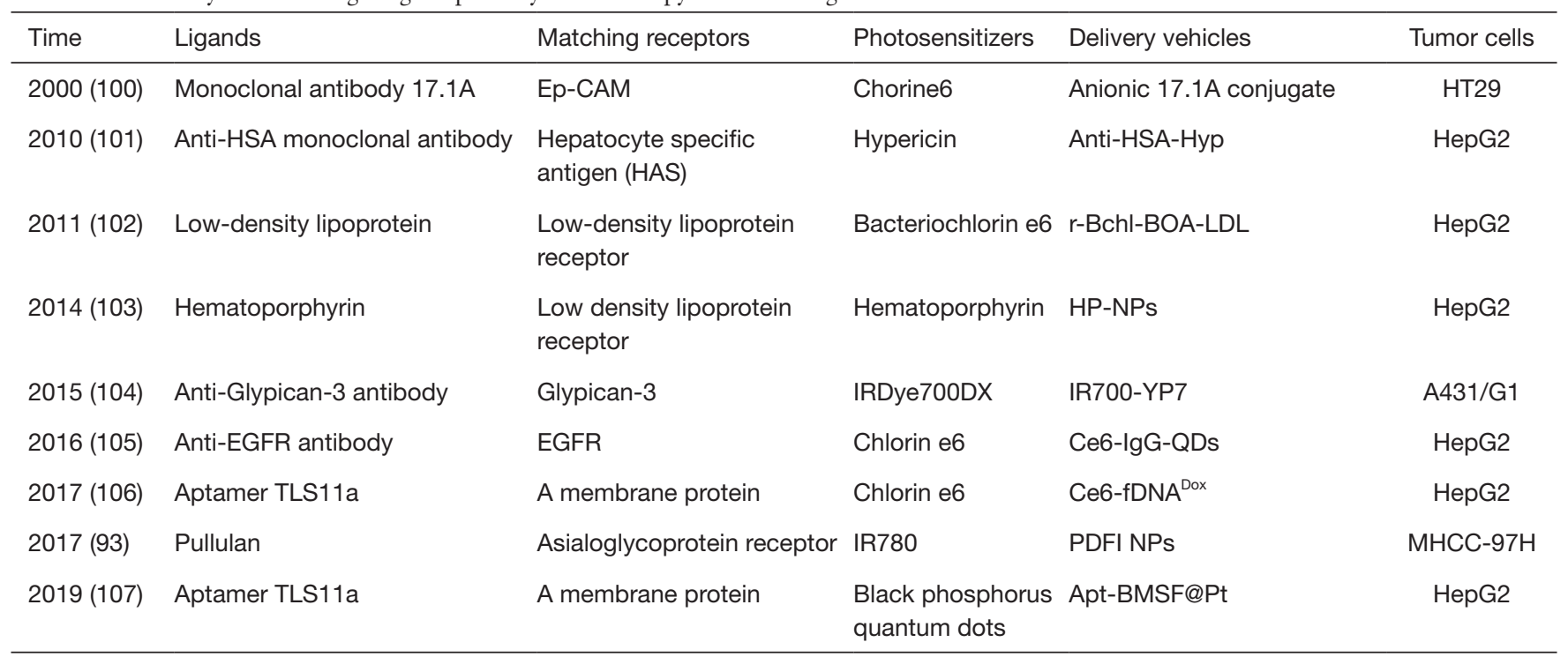

cells, pericyte deficiency, and aberrant basement membrane formation, which lead to an enhanced vascular permeability $(90,91)$. Nanocarriers (size range, 20-200 $\mathrm{nm}$ ) can extravasate and accumulate inside the interstitial space, thereby resulting in their retention in tumors (92). This passive phenomenon is called the "enhanced permeability and retention (EPR) effect". The EPR effect is now one of the most important approaches for passive targeting of PDT on tumors. A simple but effective NP system based on phospholipid, Pluronic F68 (PF68), and pullulan was designed and reported recently (93). A heptamethine dye IR780 and chemotherapeutic drug paclitaxel were loaded separately into NP system to form PDFI and PDFP NPs. In HCC cell line MHCC-97H, the combined treatment of PDFI and PDFP NPs exhibited significant synergistic effects on inhibiting cell proliferation and inducing cell apoptosis and cell cycle arrest at the G2/M phase. This study indicated that the new NP system could combine PDT with chemotherapy to treat HCC, offering a promising direction for the PDT of HCC. Iqbal et al. synthesized photosensitizers of zine-doped cobalt ferrite $\left(\mathrm{Zn}_{0.5} \mathrm{Co}_{0.5} \mathrm{Fe}_{2} \mathrm{O}_{4}\right) \mathrm{NPs}$ and $\mathrm{Co}_{3} \mathrm{O}_{4}$ nanocrystalline materials and found they could inhibit the cell viability of HepG2 cells effectively when exposed to appropriate wavelength light $(94,95)$. Recently, a new photosensitizer called coppercysteamine NPs (Cu-Cy NPs) was reported (96). Cu-Cy NPs-mediated PDT could notably inhibit HepG2 cells. The Cu-Cy NPs can enter into the exosomes secreted by tumor cells, and exosomes could be used to deliver $\mathrm{Cu}-\mathrm{Cy}$
NPs to target tumor cells.

Several proteins and molecules can be overexpressed on the surface of liver tumor cells compared with normal cells. The ligands of these proteins and molecules, including (poly)saccharides, vitamins, antibodies, peptides or other small molecules, are utilized to decorate drug delivery systems $(92,97)$. NPs or photosensitizers may exhibit tumortargeting capability by surface modification of ligands for the receptors in liver tumor tissues (98) in a process called active targeting. Active targeting can further improve the targeting property of photosensitizers toward the liver tumor tissues (99). Table 1 provides active targeting studies for the PDT of liver malignancies.

\section{Conclusion}

PDT has been applied for patients with liver cancer and has shown clinically beneficial effects. However, the liver is a special metabolic organ that possesses a remarkable capacity for regeneration. In addition, the liver features a large volume, and it is deeply located in the abdominal cavity. Difficulty arises in the development of appropriate photosensitizers, which are triggered by long-wavelength light and highly selective between normal tissues and cancerous cells in the liver. Additionally, PDT studies on humans remain inadequate, and better delivery methods for laser radiation are needed. Future research should develop new photosensitizers, such as NPs or active targeting of photosensitizers specific to liver malignancies. 
Meanwhile, the development of endoscopy-assisted PDT and IPDT is meaningful to improve the efficacy of PDT on liver malignancies. Patients receiving PDT along with other palliative methods, such as RFA, chemotherapy, or biliary stenting, can obtain better results than PDT alone. In summary, PDT can be used as a palliative treatment modality to improve the quality and duration of life of patients with liver malignancies. On this basis, PDT can play a positive role in the treatment of liver malignancies.

\section{Acknowledgments}

Funding: This study was supported by the grants from the National Natural Science Foundation of China (No. 81773293 and No. 81970569), Natural Science Foundation of Hunan Province (2019JJ50874), and Science and Technology Program of Changsha (kq1907079).

\section{Footnote}

Conflicts of Interest: The authors have no conflicts of interest to declare.

Ethical Statement: The authors are accountable for all aspects of the work in ensuring that questions related to the accuracy or integrity of any part of the work are appropriately investigated and resolved.

Open Access Statement: This is an Open Access article distributed in accordance with the Creative Commons Attribution-NonCommercial-NoDerivs 4.0 International License (CC BY-NC-ND 4.0), which permits the noncommercial replication and distribution of the article with the strict proviso that no changes or edits are made and the original work is properly cited (including links to both the formal publication through the relevant DOI and the license). See: https://creativecommons.org/licenses/by-ncnd/4.0\%.

\section{References}

1. Torre LA, Bray F, Siegel RL, et al. Global cancer statistics, 2012. CA Cancer J Clin 2015;65:87-108.

2. Bruix J, Reig M, Sherman M. Evidence-Based Diagnosis, Staging, and Treatment of Patients With Hepatocellular Carcinoma. Gastroenterology 2016;150:835-53.

3. Forner A, Llovet JM, Bruix J. Hepatocellular carcinoma. Lancet 2012;379:1245-55.
4. Li L, Wang H. Heterogeneity of liver cancer and personalized therapy. Cancer Lett 2016;379:191-7.

5. Cornu R, Beduneau A, Martin H. Influence of nanoparticles on liver tissue and hepatic functions: A review. Toxicology 2020;430:152344.

6. Iqbal S, Fakhar-e-Alam M, Akbar F, et al. Application of silver oxide nanoparticles for the treatment of cancer. Journal of Molecular Structure 2019;1189:203-9.

7. Atif M, Farooq WA, Siddiqui MA, et al. Preliminary study of spectral features of normal and malignant cell cultures. Laser Physics 2016;26.

8. Rkein AM, Ozog DM. Photodynamic therapy. Dermatol Clin 2014;32:415-25, $\mathrm{x}$.

9. Yanovsky RL, Bartenstein DW, Rogers GS, et al. Photodynamic therapy for solid tumors: A review of the literature. Photodermatol Photoimmunol Photomed 2019;35:295-303.

10. Atif M, Firdous S, Khurshid A, et al. In vitro study of 5-aminolevulinic acid-based photodynamic therapy for apoptosis in human cervical HeLa cell line. Laser Physics Letters 2009;6:886-91.

11. Atif M, Fakhar-E-Alam M, Firdous S, et al. Study of the efficacy of 5-ALA mediated photodynamic therapy on human rhabdomyosarcoma cell line (RD). Laser Physics Letters 2010;7:757-64.

12. Atif M, Firdous S, Mahmood R, et al. Cytotoxic and photocytotoxic effect of PhotofrinA (R) on human laryngeal carcinoma (Hep2c) cell line. Laser Physics 2011;21:1235-42.

13. Agostinis P, Berg K, Cengel KA, et al. Photodynamic therapy of cancer: an update. CA Cancer J Clin 2011;61:250-81.

14. Civantos FJ, Karakullukcu B, Biel M, et al. A Review of Photodynamic Therapy for Neoplasms of the Head and Neck. Adv Ther 2018;35:324-40.

15. Kwiatkowski S, Knap B, Przystupski D, et al. Photodynamic therapy - mechanisms, photosensitizers and combinations. Biomed Pharmacother 2018;106:1098-107.

16. Kim KH, Park JJ. The effects of photodynamic therapy in upper-gastrointestinal malignant diseases. Gut Liver 2010;4 Suppl 1:S39-43.

17. Maeding N, Verwanger T, Krammer B. Boosting TumorSpecific Immunity Using PDT. Cancers (Basel) 2016;8. doi: 10.3390/cancers8100091

18. Buytaert E, Dewaele M, Agostinis P. Molecular effectors of multiple cell death pathways initiated by photodynamic therapy. Biochim Biophys Acta 2007;1776:86-107.

19. Kessel D. Apoptosis and associated phenomena as a 
determinants of the efficacy of photodynamic therapy. Photochem Photobiol Sci 2015;14:1397-402.

20. Skupin-Mrugalska P, Sobotta L, Kucinska M, et al. Cellular changes, molecular pathways and the immune system following photodynamic treatment. Curr Med Chem 2014;21:4059-73.

21. Dolmans DE, Fukumura D, Jain RK. Photodynamic therapy for cancer. Nat Rev Cancer 2003;3:380-7.

22. Lange C, Bednarski PJ. Photosensitizers for Photodynamic Therapy: Photochemistry in the Service of Oncology. Curr Pharm Des 2016;22:6956-74.

23. Akram MW, Raziq F, Fakhar-e-Alam M, et al. Tailoring of $\mathrm{Au}-\mathrm{TiO} 2$ nanoparticles conjugated with doxorubicin for their synergistic response and photodynamic therapy applications. J Photochem Photobiol A Chem 2019;384:112040.

24. Seitz G, Krause R, Fuchs J, et al. In vitro photodynamic therapy in pediatric epithelial liver tumors promoted by hypericin. Oncol Rep 2008;20:1277-82.

25. Yamashita Y, Moriyasu F, Tamada T, et al. [Evaluation of the efficacy of photodynamic therapy on experimental hepatocellular carcinoma--using local injection of photosensitizer]. Nihon Gan Chiryo Gakkai Shi 1990;25:770-5.

26. Holt S, Tulip J, Hamilton D, et al. Experimental laser phototherapy of the Morris 7777 hepatoma in the rat. Hepatology 1985;5:175-80.

27. Barathan M, Mariappan V, Shankar EM, et al. Hypericinphotodynamic therapy leads to interleukin-6 secretion by HepG2 cells and their apoptosis via recruitment of $\mathrm{BH} 3$ interacting-domain death agonist and caspases. Cell Death Dis 2013;4:e697.

28. Bahng S, Yoo BC, Paik SW, et al. Photodynamic therapy for bile duct invasion of hepatocellular carcinoma. Photochem Photobiol Sci 2013;12:439-45.

29. Shi R, Li C, Jiang Z, et al. Preclinical Study of Antineoplastic Sinoporphyrin Sodium-PDT via In Vitro and In Vivo Models. Molecules 2017. doi: 10.3390/ molecules22010112.

30. Tang PM, Zhang DM, Xuan NH, et al. Photodynamic therapy inhibits $\mathrm{P}$-glycoprotein mediated multidrug resistance via JNK activation in human hepatocellular carcinoma using the photosensitizer pheophorbide a. Mol Cancer 2009;8:56.

31. Tang PM, Bui-Xuan NH, Wong CK, et al. Pheophorbide a-Mediated Photodynamic Therapy Triggers HLA Class I-Restricted Antigen Presentation in Human Hepatocellular Carcinoma. Transl Oncol 2010;3:114-22.
32. Andrzejak M, Price M, Kessel DH. Apoptotic and autophagic responses to photodynamic therapy in 1c1c7 murine hepatoma cells. Autophagy 2011;7:979-84.

33. Domagala A, Stachura J, Gabrysiak M, et al. Inhibition of autophagy sensitizes cancer cells to Photofrin-based photodynamic therapy. BMC Cancer 2018;18:210.

34. Zhang H, Ma W, Li Y. Generation of effective vaccines against liver cancer by using photodynamic therapy. Lasers Med Sci 2009;24:549-52.

35. Lee W, Han HS, Ahn S, et al. Correlation between Resection Margin and Disease Recurrence with a Restricted Cubic Spline Model in Patients with Resected Hepatocellular Carcinoma. Dig Surg 2018;35:520-31.

36. Muragaki Y, Akimoto J, Maruyama T, et al. Phase II clinical study on intraoperative photodynamic therapy with talaporfin sodium and semiconductor laser in patients with malignant brain tumors. J Neurosurg 2013;119:845-52.

37. Hu Y, Masamune K. Flexible laser endoscope for minimally invasive photodynamic diagnosis (PDD) and therapy (PDT) toward efficient tumor removal. Opt Express 2017;25:16795-812.

38. Doherty B, Nambudiri VE, Palmer WC. Update on the Diagnosis and Treatment of Cholangiocarcinoma. Curr Gastroenterol Rep 2017;19:2 .

39. Razumilava N, Gores GJ. Cholangiocarcinoma. Lancet 2014;383:2168-79.

40. Bergquist A, von Seth E. Epidemiology of cholangiocarcinoma. Best Pract Res Clin Gastroenterol 2015;29:221-32.

41. Feng JW, Yang XH, Wu BQ, et al. Progress in diagnosis and surgical treatment of perihilar cholangiocarcinoma. Gastroenterol Hepatol 2019;42:271-9.

42. Forner A, Vidili G, Rengo M, et al. Clinical presentation, diagnosis and staging of cholangiocarcinoma. Liver Int 2019;39 Suppl 1:98-107.

43. Matull WR, Dhar DK, Ayaru L, et al. R0 but not R1/R2 resection is associated with better survival than palliative photodynamic therapy in biliary tract cancer. Liver Int 2011;31:99-107.

44. Pereira SP, Aithal GP, Ragunath K, et al. Safety and long term efficacy of porfimer sodium photodynamic therapy in locally advanced biliary tract carcinoma. Photodiagnosis Photodyn Ther 2012;9:287-92.

45. Quyn AJ, Ziyaie D, Polignano FM, et al. Photodynamic therapy is associated with an improvement in survival in patients with irresectable hilar cholangiocarcinoma. HPB (Oxford) 2009;11:570-7.

46. Wiedmann M, Caca K, Berr F, et al. Neoadjuvant 
photodynamic therapy as a new approach to treating hilar cholangiocarcinoma: a phase II pilot study. Cancer 2003;97:2783-90.

47. Wagner A, Wiedmann M, Tannapfel A, et al. Neoadjuvant Down-Sizing of Hilar Cholangiocarcinoma with Photodynamic Therapy--Long-Term Outcome of a Phase II Pilot Study. Int J Mol Sci 2015;16:26619-28.

48. Ortner ME, Caca K, Berr F, et al. Successful photodynamic therapy for nonresectable cholangiocarcinoma: a randomized prospective study. Gastroenterology 2003; 125:1355-63.

49. Zoepf T, Jakobs R, Arnold JC, et al. Palliation of nonresectable bile duct cancer: improved survival after photodynamic therapy. Am J Gastroenterol 2005;100:2426-30.

50. Rizvi S, Khan SA, Hallemeier CL, et al. Cholangiocarcinoma - evolving concepts and therapeutic strategies. Nat Rev Clin Oncol 2018;15:95-111.

51. Nonaka Y, Nanashima A, Nonaka T, et al. Synergic effect of photodynamic therapy using talaporfin sodium with conventional anticancer chemotherapy for the treatment of bile duct carcinoma. J Surg Res 2013;181:234-41.

52. Park DH, Lee SS, Park SE, et al. Randomised phase II trial of photodynamic therapy plus oral fluoropyrimidine, S-1, versus photodynamic therapy alone for unresectable hilar cholangiocarcinoma. Eur J Cancer 2014;50:1259-68.

53. Hong MJ, Cheon YK, Lee EJ, et al. Long-term outcome of photodynamic therapy with systemic chemotherapy compared to photodynamic therapy alone in patients with advanced hilar cholangiocarcinoma. Gut Liver 2014;8:318-23.

54. Wentrup R, Winkelmann N, Mitroshkin A, et al. Photodynamic Therapy Plus Chemotherapy Compared with Photodynamic Therapy Alone in Hilar Nonresectable Cholangiocarcinoma. Gut Liver 2016;10:470-5.

55. Ortner MA. Photodynamic therapy for cholangiocarcinoma: overview and new developments. Curr Opin Gastroenterol 2009;25:472-6.

56. Banales JM, Cardinale V, Carpino G, et al. Expert consensus document: Cholangiocarcinoma: current knowledge and future perspectives consensus statement from the European Network for the Study of Cholangiocarcinoma (ENS-CCA). Nat Rev Gastroenterol Hepatol 2016;13:261-80.

57. Leggett CL, Gorospe EC, Murad MH, et al. Photodynamic therapy for unresectable cholangiocarcinoma: a comparative effectiveness systematic review and metaanalyses. Photodiagnosis Photodyn Ther 2012;9:189-95.
58. Lu Y, Liu L, Wu JC, et al. Efficacy and safety of photodynamic therapy for unresectable cholangiocarcinoma: A meta-analysis. Clin Res Hepatol Gastroenterol 2015;39:718-24.

59. Moole H, Tathireddy H, Dharmapuri S, et al. Success of photodynamic therapy in palliating patients with nonresectable cholangiocarcinoma: A systematic review and meta-analysis. World J Gastroenterol 2017;23:1278-88.

60. Dolak W, Schwaighofer H, Hellmich B, et al. Photodynamic therapy with polyhematoporphyrin for malignant biliary obstruction: A nationwide retrospective study of 150 consecutive applications. United European Gastroenterol J 2017;5:104-10.

61. Lee TY, Cheon YK, Shim CS. Photodynamic Therapy in Patients with Advanced Hilar Cholangiocarcinoma: Percutaneous Cholangioscopic Versus Peroral Transpapillary Approach. Photomed Laser Surg 2016;34:150-6.

62. Sato M, Inoue H, Ogawa S, et al. Limitations of percutaneous transhepatic cholangioscopy for the diagnosis of the intramural extension of bile duct carcinoma. Endoscopy 1998;30:281-8.

63. Sharma D, Subbarao G, Saxena R. Hepatoblastoma. Semin Diagn Pathol 2017;34:192-200.

64. Jendzelovska Z, Jendzelovsky R, Kucharova B, et al. Hypericin in the Light and in the Dark: Two Sides of the Same Coin. Front Plant Sci 2016;7:560.

65. Seitz G, Warmann SW, Armeanu S, et al. In vitro photodynamic therapy of childhood rhabdomyosarcoma. Int J Oncol 2007;30:615-20.

66. Goncalves J, Bernal C, Imasato H, et al. Hypericin cytotoxicity in tumor and non-tumor cell lines: A chemometric study. Photodiagnosis Photodyn Ther 2017;20:86-90.

67. Bergmann F, Stepp H, Metzger R, et al. In vitro and in vivo evaluation of photodynamic techniques for the experimental treatment of human hepatoblastoma and neuroblastoma: preliminary results. Pediatr Surg Int 2008;24:1331-3.

68. Dahl TA, Bilski P, Reszka KJ, et al. Photocytotoxicity of curcumin. Photochem Photobiol 1994;59:290-4.

69. Winter S, Tortik N, Kubin A, et al. Back to the roots: photodynamic inactivation of bacteria based on watersoluble curcumin bound to polyvinylpyrrolidone as a photosensitizer. Photochem Photobiol Sci 2013;12:1795-802.

70. Ellerkamp V, Bortel N, Schmid E, et al. Photodynamic Therapy Potentiates the Effects of Curcumin on 
Pediatric Epithelial Liver Tumor Cells. Anticancer Res 2016;36:3363-72.

71. Zsiros J, Brugieres L, Brock P, et al. Dose-dense cisplatinbased chemotherapy and surgery for children with highrisk hepatoblastoma (SIOPEL-4): a prospective, singlearm, feasibility study. Lancet Oncol 2013;14:834-42.

72. Xu J, Fan J, Qin X, et al. Chinese guidelines for the diagnosis and comprehensive treatment of colorectal liver metastases (version 2018). J Cancer Res Clin Oncol 2019;145:725-36.

73. Taniai N, Akimaru K, Yoshida H, et al. Surgical treatment for better prognosis of patients with liver metastases from colorectal cancer. Hepatogastroenterology 2007;54:1805-9.

74. Rovers JP, Saarnak AE, Molina A, et al. Effective treatment of liver metastases with photodynamic therapy, using the second-generation photosensitizer metatetra(hydroxyphenyl)chlorin (mTHPC), in a rat model. Br J Cancer 1999;81:600-8.

75. van Duijnhoven FH, Rovers JP, Engelmann K, et al. Photodynamic therapy with 5,10,15,20-tetrakis(mhydroxyphenyl) bacteriochlorin for colorectal liver metastases is safe and feasible: results from a phase I study. Ann Surg Oncol 2005;12:808-16.

76. Shafirstein G, Bellnier D, Oakley E, et al. Interstitial Photodynamic Therapy-A Focused Review. Cancers (Basel) 2017;9. doi: 10.3390/cancers9020012.

77. Vogl TJ, Eichler K, Mack MG, et al. Interstitial photodynamic laser therapy in interventional oncology. Eur Radiol 2004;14:1063-73.

78. Rovers JP, Schuitmaker JJ, Vahrmeijer AL, et al. Interstitial photodynamic therapy with the second-generation photosensitizer bacteriochlorin a in a rat model for liver metastases. Br J Cancer 1998;77:2098-103.

79. van Hillegersberg R, Marijnissen JP, Kort WJ, et al. Interstitial photodynamic therapy in a rat liver metastasis model. Br J Cancer 1992;66:1005-14.

80. Gomes A, Neves M, Cavaleiro JAS. Cancer, Photodynamic Therapy and Porphyrin-Type Derivatives. An Acad Bras Cienc 2018;90:993-1026.

81. Mallidi S, Anbil S, Bulin AL, et al. Beyond the Barriers of Light Penetration: Strategies, Perspectives and Possibilities for Photodynamic Therapy. Theranostics 2016;6:2458-87.

82. Yao M, Ma L, Li L, et al. A New Modality for Cancer Treatment--Nanoparticle Mediated Microwave Induced Photodynamic Therapy. J Biomed Nanotechnol 2016;12:1835-51.

83. Idris NM, Jayakumar MK, Bansal A, et al. Upconversion nanoparticles as versatile light nanotransducers for photoactivation applications. Chem Soc Rev 2015;44:1449-78.

84. Liang L, Lu Y, Zhang R, et al. Deep-penetrating photodynamic therapy with KillerRed mediated by upconversion nanoparticles. Acta Biomater 2017;51:461-70.

85. Zou X, Yao M, Ma L, et al. X-ray-induced nanoparticlebased photodynamic therapy of cancer. Nanomedicine (Lond) 2014;9:2339-51.

86. Nishiwaki Y, Nakamura S, Sakaguchi S. New method of photosensitizer accumulation for photodynamic therapy in an experimental liver tumor. Lasers Surg Med 1989;9:254-63.

87. Svanberg K, Liu DL, Wang I, et al. Photodynamic therapy using intravenous delta-aminolaevulinic acid-induced protoporphyrin IX sensitisation in experimental hepatic tumours in rats. Br J Cancer 1996;74:1526-33.

88. Kaneko J, Inagaki Y, Ishizawa T, et al. Photodynamic therapy for human hepatoma-cell-line tumors utilizing biliary excretion properties of indocyanine green. J Gastroenterol 2014;49:110-6.

89. Malatesti N, Munitic I, Jurak I. Porphyrin-based cationic amphiphilic photosensitisers as potential anticancer, antimicrobial and immunosuppressive agents. Biophys Rev 2017;9:149-68.

90. Danhier F, Feron O, Preat V. To exploit the tumor microenvironment: Passive and active tumor targeting of nanocarriers for anti-cancer drug delivery. J Control Release 2010;148:135-46.

91. Narang AS, Varia S. Role of tumor vascular architecture in drug delivery. Adv Drug Deliv Rev 2011;63:640-58.

92. Li M, Zhang W, Wang B, et al. Ligand-based targeted therapy: a novel strategy for hepatocellular carcinoma. Int J Nanomedicine 2016;11:5645-69.

93. Wang D, Zhang S, Zhang T, et al. Pullulan-coated phospholipid and Pluronic F68 complex nanoparticles for carrying IR780 and paclitaxel to treat hepatocellular carcinoma by combining photothermal therapy/ photodynamic therapy and chemotherapy. Int J Nanomedicine 2017;12:8649-70.

94. Iqbal S, Fakhar-e-Alam M, Atif M, et al. Structural, morphological, antimicrobial, and in vitro photodynamic therapeutic assessments of novel $\mathrm{Zn}+2$-substituted cobalt ferrite nanoparticles. Results Phys 2019;15:102529.

95. Iqbal S, Fakhar-e-Alam M, Atif M, et al. Photodynamic therapy, facile synthesis, and effect of sintering temperature on the structure, morphology, optical properties, and 
anticancer activity of $\mathrm{Co} 3 \mathrm{O} 4$ nanocrystalline materials in the HepG2 cell line. J Photochem Photobiol A Chem 2020;386:112130.

96. Huang X, Wan F, Ma L, et al. Investigation of coppercysteamine nanoparticles as a new photosensitizer for anti-hepatocellular carcinoma. Cancer Biol Ther 2019;20:812-25.

97. Zhong YA, Meng FH, Deng C, et al. Ligand-Directed Active Tumor-Targeting Polymeric Nanoparticles for Cancer Chemotherapy. Biomacromolecules 2014;15:1955-69.

98. Ogawara KI, Higaki K. Nanoparticle-Based Photodynamic Therapy: Current Status and Future Application to Improve Outcomes of Cancer Treatment. Chem Pharm Bull (Tokyo) 2017;65:637-41.

99. Yameen B, Choi WI, Vilos C, et al. Insight into nanoparticle cellular uptake and intracellular targeting. J Control Release 2014;190:485-99.

100. Hamblin MR, Del Governatore M, Rizvi I, et al. Biodistribution of charged 17.1A photoimmunoconjugates in a murine model of hepatic metastasis of colorectal cancer. Br J Cancer 2000;83:1544-51.

101.Fadel M, Kassab K, Youssef T. Photodynamic efficacy of hypericin targeted by two delivery techniques to hepatocellular carcinoma cells. Lasers Med Sci 2010;25:675-83

102. Marotta DE, Cao W, Wileyto EP, et al. Evaluation of

Cite this article as: Zou H, Wang F, Zhou JJ, Liu X, He Q, Wang C, Zheng YW, Wen Y, Xiong L. Application of photodynamic therapy for liver malignancies. J Gastrointest Oncol 2020;11(2):431-442. doi: 10.21037/jgo.2020.02.10 bacteriochlorophyll-reconstituted low-density lipoprotein nanoparticles for photodynamic therapy efficacy in vivo. Nanomedicine (Lond) 2011;6:475-87.

103. Chang JE, Yoon IS, Sun PL, et al. Anticancer efficacy of photodynamic therapy with hematoporphyrin-modified, doxorubicin-loaded nanoparticles in liver cancer. J Photochem Photobiol B 2014;140:49-56.

104. Hanaoka H, Nakajima T, Sato K, et al. Photoimmunotherapy of hepatocellular carcinomatargeting Glypican-3 combined with nanosized albuminbound paclitaxel. Nanomedicine (Lond) 2015;10:1139-47.

105. Yan ZY, Wang LL, Fei MY, et al. Construction of photodynamic-effect immunofluorescence probes by a complex of quantum dots, immunoglobulin $\mathrm{G}$ and chlorin e6 and their application in HepG2 cell killing. Luminescence 2016;31:1174-81.

106.Zhang D, Zheng A, Li J, et al. Tumor Microenvironment Activable Self-Assembled DNA Hybrids for $\mathrm{pH}$ and Redox Dual-Responsive Chemotherapy/PDT Treatment of Hepatocellular Carcinoma. Adv Sci (Weinh) 2017;4:1600460.

107.Lan S, Lin Z, Zhang D, et al. Photocatalysis Enhancement for Programmable Killing of Hepatocellular Carcinoma through Self-Compensation Mechanisms Based on Black Phosphorus Quantum-Dot-Hybridized Nanocatalysts. ACS Appl Mater Interfaces 2019;11:9804-13. 